\title{
BMJ Global Health How health systems approached respiratory viral pandemics over time: a systematic review
}

\author{
Fidelia Cascini (D) , Ilda Hoxhaj, Drieda Zaçe, Margherita Ferranti, \\ Maria Luisa Di Pietro, Stefania Boccia, Walter Ricciardi
}

To cite: Cascini F, Hoxhaj I, Zaçe D, et al. How health systems approached respiratory viral pandemics over time: a systematic review. BMJ Global Health 2020;5:e003677. doi:10.1136/ bmjgh-2020-003677

Handling editor Seye Abimbola

- Additional material is published online only. To view please visit the journal online (http://dx.doi.org/10.1136/ bmjgh-2020-003677).

Received 13 August 2020 Revised 26 November 2020 Accepted 3 December 2020

Check for updates

C Author(s) (or their employer(s)) 2021. Re-use permitted under CC BY-NC. No commercial re-use. See rights and permissions. Published by BMJ.

Public Health Section, Università Cattolica del Sacro Cuore Facoltà di Medicina e Chirurgia, Roma, Italy

Correspondence to Dr Fidelia Cascini; fidelia.cascini1@unicatt.it

\section{ABSTRACT}

Background Several healthcare systems facing respiratory viral infections outbreaks, like COVID-19, have not been prepared to manage them. Public health mitigation solutions ranging from isolation of infected or suspected cases to implementation of national lockdowns have proven their effectiveness for the outbreak's control. However, the adjustment of public health measures is crucial during transition phases to avoid new outbreaks. To address the need for designing evidence-based strategies, we performed a systematic review to identify healthcare systems interventions, experiences and recommendations that have been used to manage different respiratory viral infections outbreaks in the past.

Methods PubMed, Web of Science, Scopus and Cochrane were searched to retrieve eligible studies of any study design, published in English until 17 April 2020. Doubleblinded screening process was conducted by titles/ abstracts and subsequently eligible full texts were read and pertinent data were extracted. When applicable, quality assessment was conducted for the included articles. We performed a narrative synthesis of each implemented public health approaches.

Results We included a total of 24 articles addressing the public health approaches implemented for respiratory viral infections outbreaks for COVID-19, influenza A H1N1, MERS and severe acute respiratory syndrome The identified approaches are ascribable to two main categories: healthcare system strategies and healthcare provider interventions. The key components of an effective response on respiratory viral outbreaks included the implementation of evidence-based contextual policies, intrahospital management actions, community healthcare facilities, non-pharmaceutical interventions, enhanced surveillance, workplace preventive measures, mental health interventions and communication plans.

Conclusion The identified healthcare system strategies applied worldwide to face epidemics or pandemics are a useful knowledge base to inform decision-makers about control measures to be used in the transition phases of COVID-19 and beyond.

\section{INTRODUCTION}

Health systems are currently facing a new respiratory virus pandemic caused by the

\section{Key questions}

What is already known?

- Respiratory viral epidemics are a huge challenge for nations and their health systems worldwide.

- Health systems' responses to pandemics have often been managed as isolated experiences and related strategies that were adopted were considered to be context-specific.

What are the new findings?

- Twenty-four articles revealed a wide range of possible solutions that can be adopted to face pandemics.

- A comparative evaluation of non-pharmaceutical interventions remains a major controversial issue.

- There is a need for primary data to guide the structuring and implementation of strategies.

What do the new findings imply?

- International cooperation, transparency of data, connections between health experts and governmental officials are needed to support nations' management systems.

- National and local administrations should coordinate closely and be interconnected to promote a unified leadership and a hierarchical responsibility.

- Detailed reporting would be required from the scientific community to help the improvement of strategic actions.

novel coronavirus SARS-CoV-2, which emerged in December 2019 in Wuhan, China and has spread rapidly all over the world. This pandemic has revealed the weaknesses and unpreparedness of most health systems. ${ }^{1}$ WHO declared the pandemic on 11 March $2020^{2}$ and as of 27 July 2020 more than 16000000 people have been infected and over 646000 deaths have been reported worldwide in more than 200 countries. $^{3}$

As a result from the current pandemic, public health experts and government representatives have wondered how to undertake effective public health measures and restriction policies and their attempts have ranged from the isolation of infected individuals to 
the implementation of national lockdowns. The imposed lockdown strategies were effective in controlling the outbreak and resulted in a sustained reduction in the number of new cases across the world. ${ }^{4-7}$ While several countries introduced the easing of lockdowns in the summer of 2020, the planning and adjustment of public health measures at a national and international level are of utmost importance to ultimately reach and maintain a state of low level or no transmission. ${ }^{8}$ It is likely that the transition period of the COVID-19 pandemic will last several months and the implementation and constant surveillance of public health strategies are fundamental prerequisites for the organisation and management of healthcare systems. ${ }^{9}$

To look at the diverse strategies enacted to slow down and stop transmission of previously experienced pandemics-caused by other respiratory viruses such as severe acute respiratory syndrome (SARS), Middle-East Respiratory Syndrome Coronavirus, influenza A H1N1 and Influenza Pandemic, 1918-1919-could be an opportunity to better manage emerging viral outbreaks. Lessons from the past show where healthcare systems have been successful and where they have failed while coping with respiratory virus outbreaks. Furthermore, they highlight where evidence-based research is needed so that it can assist decision-makers and stakeholders in addressing challenges that emerge or are related to epidemic transition phases, including COVID-19.

In this systematic review, we aim to identify key issues, context and applications of intervention strategies that have been implemented to control emerging outbreaks as well as to summarise international, national, and local health systems' approaches that have been used to contain epidemics or pandemics and that may be currently useful as control measures in the transition phases of the COVID-19 pandemic.

\section{METHODS}

This systematic review was conducted and reported according to the Preferred Reporting Items for Systematic Reviews and Meta-Analyses statement. ${ }^{10}$ The review protocol was published in PROSPERO with registration number CRD42020194318.

\section{Search strategy}

The electronic databases of Web of Science, PubMed, Scopus and Cochrane were searched to retrieve potential eligible articles, published from the inception until 17 April 2020. A search string for PubMed was developed consisting of Medical Subject Headings terms and keywords combined by using Boolean Operators. The search in PubMed was restricted to only humans. Keywords such as "Pandemics"; "Epidemics"; "SARS"; "MERS"; "COVID-19"; "Influenza Pandemic, 19181919"; "Strategies"; "Management"; "Surveillance" were used. The search string was adapted for use in the other electronic databases. The full search strategy for all databases can be found in online supplemental material 1 .

\section{Inclusion/exclusion criteria}

We included articles that discussed healthcare system strategies or healthcare provider interventions that have been used to manage respiratory viral epidemics or pandemics, with any study design (quantitative or qualitative) and that are published in English. We excluded systematic reviews, non-empirical studies, conference abstracts, editorials, commentaries, book reviews and abstracts not accompanied by a full text. Furthermore, animal and modelling studies were excluded. We aimed to collect and summarise information concerning health systems' approaches, from both a broad and holistic point of view, which addressed healthcare challenges during respiratory virus outbreaks. We also excluded studies discussing clinical therapeutics; clinical epidemiological data; vaccines; knowledge; behaviours and education on pandemics; economic studies; and restrictions concerning school closure, travel and transportation. The included articles were grouped into two main categories: (1) articles reporting approaches related to healthcare providers and (2) articles reporting approaches related to healthcare systems. For the purposes of this review, the term 'healthcare system strategies' refers to the strategy of any health system which provides leadership, management and direction to healthcare units through a set of policies and plans adopted by government, private sector and other groups in areas such as financing, human resources and public health. The term 'healthcare provider management' refers to any organisation or institution delivering healthcare services to the population.

\section{Study selection}

All studies retrieved from the search strategy were imported to Mendeley and duplicates were removed. The remaining studies were uploaded to RAYYAN QCRI software. Three researchers (MF, IH and DZ) independently performed the first screening based on titles and abstracts. In a second step, studies with full texts available were entirely read by the researchers to decide the final articles to include in the review. Disagreements were resolved through discussion between the review team members. The reference lists of the included studies were hand searched to look for additional studies.

\section{Data extraction and synthesis}

Data extraction was performed by three researchers (MF, $\mathrm{IH}$ and DZ). A dedicated data extraction form was used to retrieve the following information for each eligible study:

1. Study identification (first author, title, publication year).

2. Study characteristics (period, country, design, disease, population).

3. Healthcare approach categories (ie, healthcare systems strategies and healthcare providers management). 
4. Healthcare approach subcategories:

1. About healthcare system strategies: workplace and university measures, non-pharmaceutical interventions (NPIs), communication strategies, healthcare policy development, mental health interventions and epidemiological surveillance.

2. About healthcare provider management: hospitals and community healthcare facilities.

5. Description of the implemented approach (focus of the intervention, design and key components, tools used to implement it, stakeholder group, setting).

6. Main findings and possible outcomes of the implemented approach.

We developed a synthetic narrative analysis, matching and grouping studies according to the type of approach implemented and described by each study, then organised the information into local categories and subcategories. This resulted in classifying studies that reported healthcare system strategies into six subcategories based on their approaches to address healthcare challenges during respiratory virus outbreaks and classifying studies reporting healthcare provider management interventions into two subcategories. Findings related to each article's topic were analysed and synthesised to determine the most important and most used approaches as well as gaps in knowledge, to be able to drive to conclusions in terms of their implications for public health and governmental decision-makers in responding to the COVID-19 pandemic transition phases.

\section{Quality assessment}

When appropriate, two researchers (IH and MF) independently conducted the methodological quality assessment, based on the study designs. Disagreements were resolved by discussion with a third researcher (DZ). The standardised critical appraisal tool was the Joanna Briggs Institute Qualitative Assessment and Review Instrument and it was used to assess the methodological quality of included papers. ${ }^{11}$ This tool offers the possibility to appraise the methodological quality of articles based on the satisfaction or not of several specific criteria for each study design. To assess the quality of the narrative reviews included in the systematic review we used the Scale for the Assessment of Narrative Review Articles ${ }^{12}$ and the 'Study Quality Assessment Tools' of the National Heart, Lung and Blood Institute for the before-after study. ${ }^{13}$ The latter gives six criteria to evaluate on a scale from 0 to 2 , where 0 is appointed when the specific criteria is not satisfied at all and two when the criteria is completely satisfied. To summarise the overall evidence quality, we grouped the articles into three categories: good (studies met at least $75 \%$ of the quality criteria), moderate (studies met between $50 \%$ and $74 \%$ of the quality criteria) and poor (studies met less than $50 \%$ of the quality criteria) methodological quality.

\section{Patient and public involvement}

Patients and the public were not involved in any way in this study which is a systematic review of the literature.

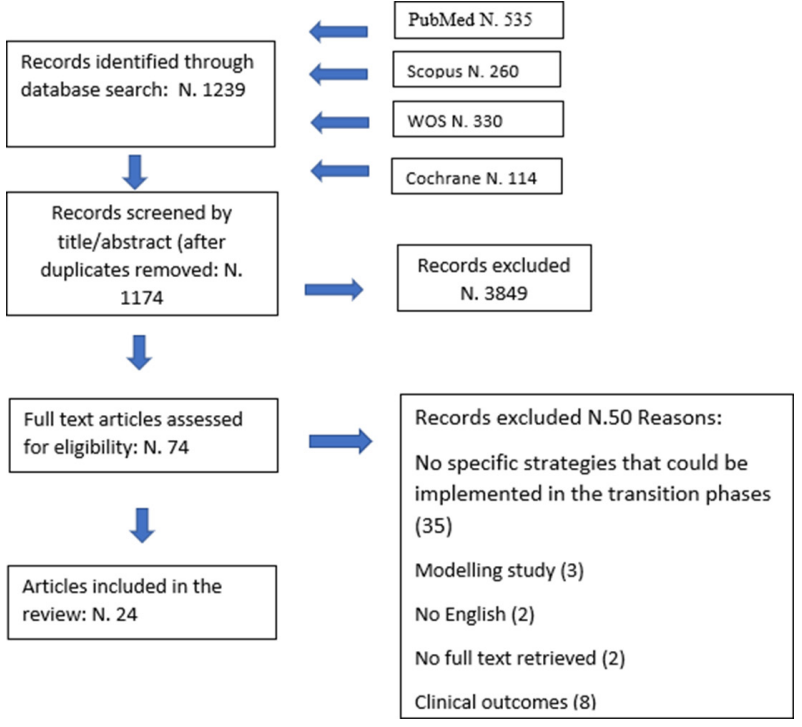

Figure 1 The process of study screening and selection.

\section{RESULTS}

\section{Results of the search strategy}

A total of 1239 articles were retrieved from all databases. After the screening process, 24 articles ${ }^{14-37}$ satisfied the inclusion criteria and were included in the systematic review. No further studies were retrieved from the handsearch of the reference lists of the included studies. The process of study screening and selection is reported in figure 1 .

\section{Results of the quality assessment}

The majority of articles $(58.3 \%)^{14151923-252729303235-38}$ had a specific study design, while the rest $(41.7 \%)^{16-1820-2226283134}$ were literature reviews or guidelines without a designated study design, so it was not applicable to perform a quality assessment for the latter reviews. Among the articles for which we conducted the quality assessment (14 studies), ${ }^{14} 151923-2527293032$ 35-38 $78 \%$ had a good quality (satisfied $75 \%$ or more of the quality criteria), while the rest $(25 \%)$ had a moderate quality (satisfied between $50 \%$ and $74 \%$ of quality criteria). The quality assessment of the included articles is reported in online supplemental material 2, according to the quality appraisal tool used.

\section{Characteristics of the included studies}

Twelve ${ }^{14-16} 1819263032-3537$ articles reported data on influenza A H1N1, seven ${ }^{21-25} 2728$ articles were on COVID-19, one article ${ }^{29}$ was on MERS, one article was on SARS, ${ }^{20}$ two articles ${ }^{1736}$ were on both influenza A H1N1 and SARS and one article ${ }^{31}$ was on influenza A H1N1, SARS and MERS. The included articles were published from 2008 until 2020. Regarding the study design, the majority $(20.8 \%)$ were case reports, followed by qualitative articles $(12.5 \%)$, narrative reviews $(8.3 \%)$ and cohort, cross sectional, before-and-after and randomised clinical trials, each with only one article, respectively. $37.4 \%$ of the articles did not have a specific study design or a designated methodological approach and were reported as 
descriptive literature reviews. The included articles were grouped in two main categories: (1) regarding the strategies adopted to face the outbreaks at a broad, national level and named healthcare system strategies and (2) concerning the plans adopted to manage the outbreaks at a healthcare provider level and named healthcare providers management.

\section{Healthcare system strategies}

Several articles ${ }^{14} 1521263132$ reported on the healthcare system strategies used in facing the emergencies related to the epidemics. The adopted strategies belong to the following subcategories: healthcare policies (seven articles), ${ }^{16} 172230333637$ communication strategies (eight articles), ${ }^{20} 22263033-36$ NPIs (three articles), ${ }^{26} 3132$ epidemiological surveillance strategies (three articles), ${ }^{20} 3134$ mental health interventions (four articles) ${ }^{21} 222528$ and workplace and university measures (three articles). ${ }^{141521}$ Several articles referred to more than one subcategory, indicating that the combination of these strategies would provide a synergic and more effective action (table 1).

\section{Healthcare policy development}

Seven articles ${ }^{161722} 30333637$ on pandemic management policies suggested that policy implementation should be supported by evidence-based decisions and be based on countries' past experiences. Six articles reported the policy development lessons learnt from influenza A H1N1, two articles from SARS and influenza A H1N1and one article from COVID-19. All articles concluded that policy planning and development should enable the realisation of a coordinated response, whereas the other components to be considered during the development differed according to the healthcare system capacity. Lessons learnt from Australia during the 2009 influenza A H1N1 pandemic suggested that the decision-making and communication processes should be intimately linked with each other, better coordinated at a national and regional level and aligned with the experts' advice.

Specifically, it was recommended to focus on the transition phase of the epidemic through the following ways:

1. To realise a careful downscaling of response as the pandemic is brought under control.

2. To recover and restore the healthcare system and return to the alert phase as quickly as possible.

3. To enhance the vigilance for possible subsequent waves.

4. To increase the monitoring plans to control the virus transmission. ${ }^{16}$

Policy planning and development, as based on New Zealand's experience with influenza A H1N1, should consider the following actions:

1. The realisation of a coordinated response and its communication.

2. The development of a combined public health/primary care response during the 'containment' phase.
3. The creation of a universal red/green stream during the 'manage it' phase of the epidemic supported by dedicated influenza centres and call centres.

According to the recommendations of healthcare service workers in Canada, based on their encountered barriers during influenza A H1N1, policies for future pandemics should include:

1. The distribution of supplies in a timely fashion.

2. The establishment of an emergency fund.

3 . The involvement of more human resources.

4. The deployment of an interdisciplinary team of healthcare professionals and the general community.

5 . The increase of community awareness regarding disease processes and prevention.

6 . The opening of an 'alternative care site' as a satellite centre providing necessary health services. ${ }^{37}$

Considering the weaknesses of a health system's response, such as those reported in Mexico during influenza A H1N1, policy plans should incorporate:

1. The cooperation between different countries.

2. The promotion of information exchange.

3 . The creation of international governmental networks. ${ }^{30}$

The development of pandemic preparedness and response guidelines, along with a well-planned, effectively communicated and coordinated emergency response, were considered to be the key strategy components for influenza A H1N1 and SARS management. ${ }^{17}$ The key components of a pandemic management policy, considering the Hazard Analysis and Critical Control Points (HACCP) analysis on their weaknesses and strengths during influenza A H1N1 and SARS, were the following:

1. To identify and name the key operators in all institutions involved in outbreak management.

2. To develop specific guidelines.

3. To harmonise national, regional and institutional epidemic plans.

4. To capacitate surveillance databases and to create systems to analyse huge datasets.

5. To develop strategies to cope with limited resources during the outbreaks. ${ }^{36}$

The development of pandemic policies, according to the Strengths, Weaknesses, Opportunities and Threats (SWOT) analysis on China's response during COVID-19, should be people-oriented and continuously reshaped to answer to the global public crisis. The highest priority areas to be addressed in the policies were recognised as:

1. The continuous evolution of an emergency health system.

2. The strengthening of international and domestic linkages and public intervention in responding to public health emergencies.

3. The enhancement of related scientific research.

4. The development of a plan for an infectious disease reporting system.

5. The formulation of a return-to-work plan for different industries. $^{22}$ 


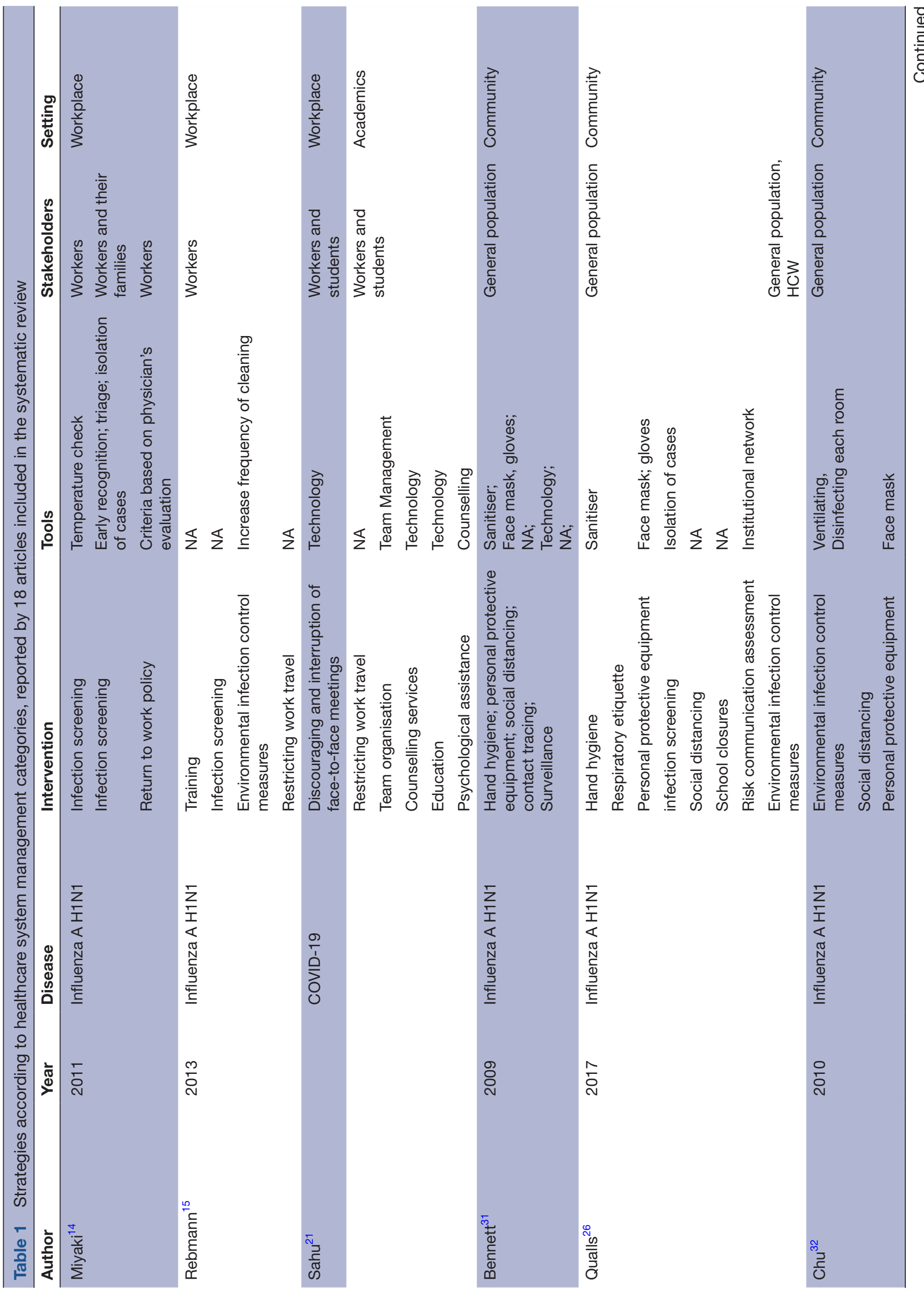

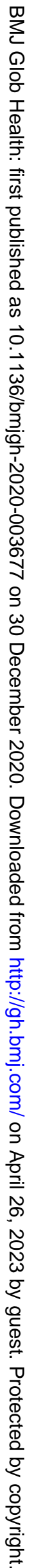




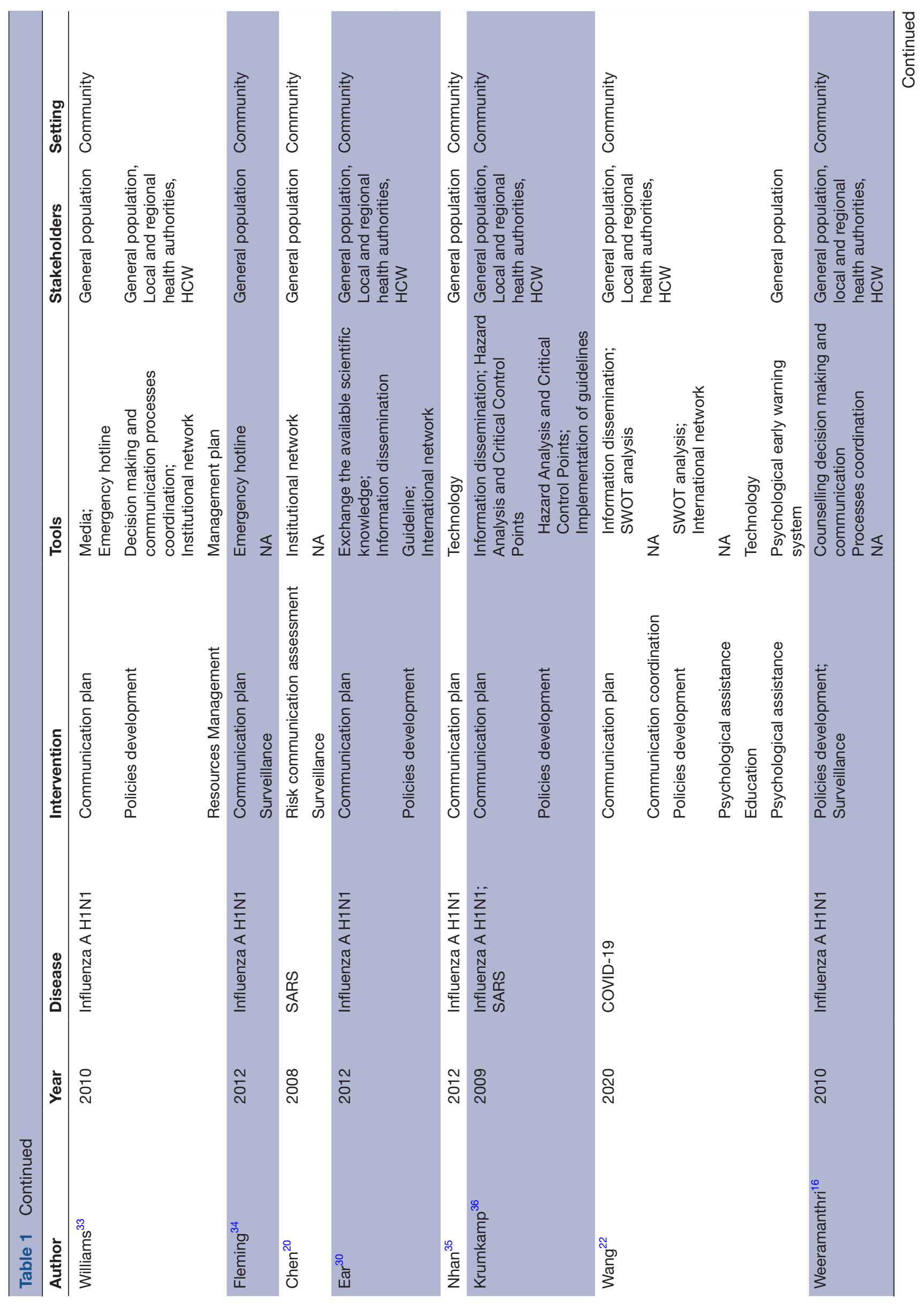

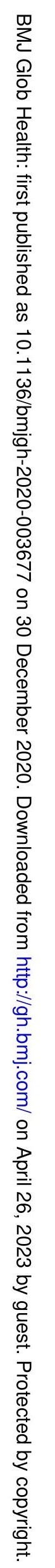




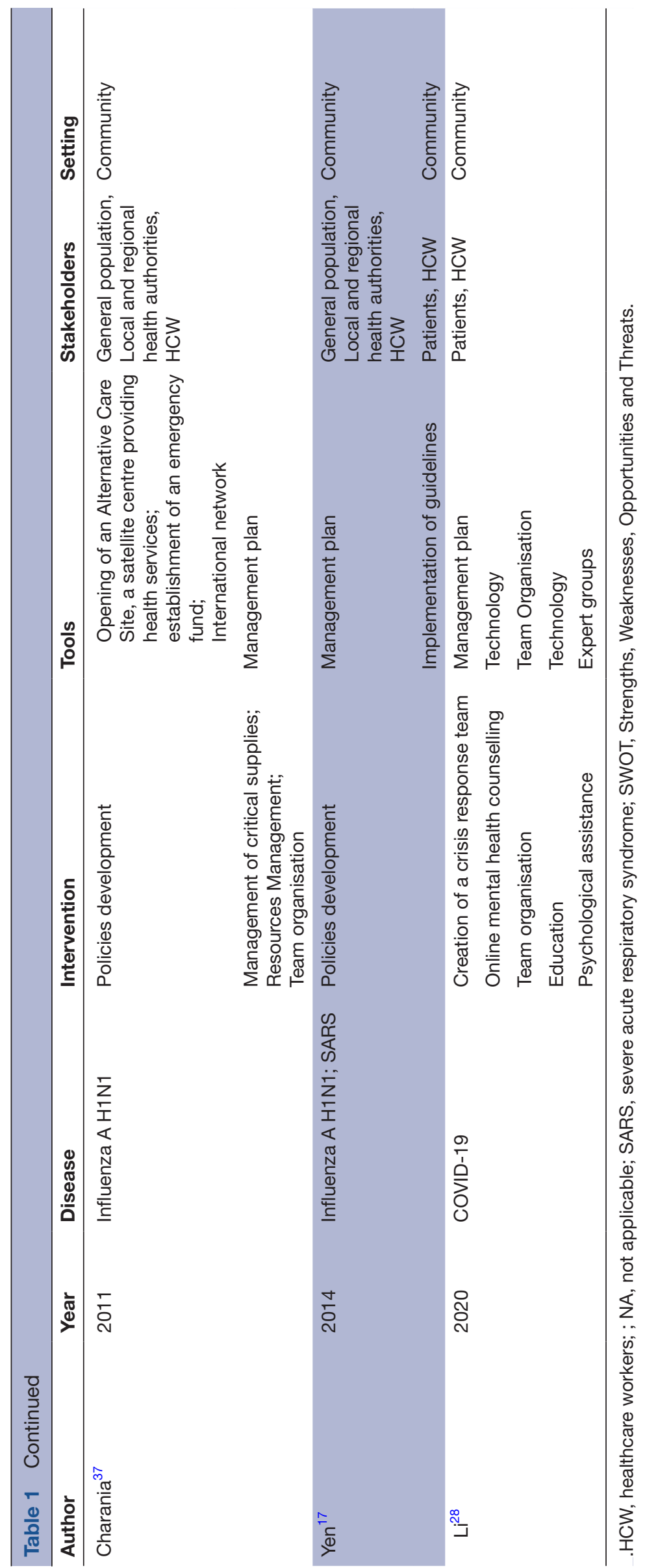

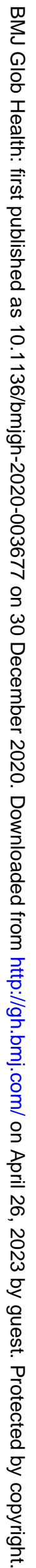




\section{Communication strategies}

Eight articles $2022263033-36$ underlined the need for an effective communication strategy about the risk related to the diffusion of the disease's outbreak. Risk communication strategies during the influenza A H1N1 outbreak in the USA were considered important because they allowed people to ponder the need for trust in the government to induce people to comply with the regulations. ${ }^{26}$ Finding the best way to inform people about the correct use of personal NPI and to provide medical advice for patients with influenza-like-symptoms was considered a critical aspect during the influenza A H1N1 outbreaks in New Zealand ${ }^{33}$ and England. ${ }^{34}$ To cope with pressures on primary care, the communication strategy included a dedicated emergency telephone-based facility and illustrated messages transferred via media. The need for a clear and well-defined communication strategy, among both the public and healthcare providers, also emerged during the influenza A H1N1 outbreaks in Mexico ${ }^{30}$ and Canada, ${ }^{35}$ where technology was considered important as a way to improve the coordination of the communication plan and avoid the variability and incongruities in developing guidelines. An effective communication strategy, to both the stakeholders and the general public, was also deemed important for a better public participation and support to contain also SARS. ${ }^{20}$ A HACCP analysis assessed the policies applied to face the influenza A H1N1 and SARS outbreak in European countries. It underlined the importance of a communication strategy that involves all levels of pandemic management, including institutions that have the responsibilities of administrating and coordinating the response as well as disseminating information to healthcare providers who are taking part in the management. ${ }^{36}$ In a similar way, the SWOT analysis (Strengths (S), Weaknesses (W), Opportunities (O) and Threats $(\mathrm{T})$ ) on China's response towards COVID-19 led to criticisms about proper communication among all of the stakeholders involved in the management of epidemics, including institutions, international organisations and governments, who are in charge of coordinating the control strategy. ${ }^{22}$

\section{Non-pharmaceutical interventions}

Three articles ${ }^{26} 3132$ considered NPIs as a useful tool to reduce the spread of the respiratory viral infections while waiting for the availability of vaccines and specific therapies and concluded that the use of a single NPI is only partially effective. The effectiveness of multiple NPIs during the influenza A H1N1 pandemic in the USA involved personal (voluntary home isolation, respiratory etiquette, hand hygiene), community (social distancing measures, school closures and dismissals) and environmental (surface cleaning measures) ${ }^{26}$ aspects. The combination of different NPIs included hand hygiene; the use of appropriate and well-fitted facemasks; ventilation and disinfection of each room; and isolation of suspected cases. Additionally, enhanced surveillance, contact tracing and a quarantine for asymptomatic contacts, were effective tools in preventing a secondary outbreak of the 2009 influenza A H1N1 in China ${ }^{32}$ and in containing the spread of influenza A H1N1 and SARS. ${ }^{31}$ Considering the reported effectiveness of NPIs in controlling the spread of respiratory viruses, the continuity of these measures throughout the epidemics' transition phases was highly recommended.

\section{Epidemiological surveillance}

Three articles ${ }^{20} 3134$ reported that rapid and early identification of positive cases during respiratory viral epidemics allow healthcare systems to put prompt control measures in place to prevent the spread of the infection. In Taiwan, contact tracing strategies during the SARS outbreak were used to rapidly identify possible early secondary cases and any unrecognised source of infection for persons without epidemiological links, along with a quarantine of asymptomatic contacts proved effective in containing the infection. ${ }^{20}$ The enhanced surveillance was crucial in increasing the capability for effective control measures to contain and limit the spread of influenza A H1N1 in Australia ${ }^{31}$ and England as well. ${ }^{34}$ The latter highlighted the importance of an appropriate regional-based surveillance programme, operating throughout the year in summer and in winter. ${ }^{34}$

\section{Mental health interventions}

Four articles 21222528 reported the integration of psychological crisis intervention into the general deployment of disease prevention during COVID-19 in China, the USA and Jamaica. Mental health national associations were required to implement interventions and to create psychological assistance groups of experts to provide professional guidance and coordination with the health authorities. According to the 'Principles for Emergency Psychological Crisis Intervention for COVID-19' issued by the Chinese authorities, ${ }^{28}$ the interventions related to mental health should address three main points: (1) understanding the mental health status in the different populations involved in the COVID-19 outbreaks; (2) identifying people who are at high risk of suicide and aggression; (3) providing appropriate psychological interventions for those who need it. Specific measures, such as the creation of a crisis response team that provides online mental health counselling and education for both patients and front-line medical staff, should also be adopted widely at a national level. The establishment of a social psychological early warning system, the strengthening of a public psychological health education system and the provision of psychological counselling, for the general public, ${ }^{22}$ and in particular for students, ${ }^{21}$ has been considered to be of utmost importance in reducing the risk of negative psychological outcomes. Based on previously experienced 
pandemics, a fundamental mental health intervention is the 'psychological first aid' tool, which can mitigate the acute psychological distress and offer a compassionate and supportive presence to continuously assess the healthcare workers' needs. ${ }^{25}$

\section{Workplace and university measures}

Three articles ${ }^{141521}$ evaluated the preventive measures implemented in non-healthcare workplaces and their effectiveness, especially during influenza A H1N1 and COVID-19. A randomised controlled trial conducted in Japan ${ }^{14}$ reported that the adoption of combined workplace measures reduced the overall risk for influenza A H1N1 infection transmission by $20 \%$ in the workplace. These measures included: the measurements of body temperature before leaving work each day; the reporting of any symptoms of influenza-like illness among workers or their family members; the obligation to stay at home for the symptomatic worker; and the possibility to return to the workplace based on specific criteria and after physician evaluation. To minimise the influenza A H1N1 disease, healthcare and non-healthcare businesses in USA implemented, based on Centers for Disease Control and Prevention (CDC) recommendations, the following actions: (1) the training of employees on ifluenza A H1N1 disease; (2) the promotion of respiratory hygiene practices; (3) the availability of free medical consultations for the staff; (4) the availability of screening practice for the illness; (5) the higher frequency and intensity of environmental cleaning; (6) the discouraging of faceto-face meetings and (7) the restriction of business travel. However, the implementation of these measures was affected by factors associated with the size of the company involved and if this company was related to a healthcare business or not. ${ }^{15}$ Preventive measures should also be implemented in academic workplaces, which especially faced challenges during COVID-19 due to the interruption of face-to-face lessons. Going through the transition phases of a pandemic, it was suggested that a task force with a multidisciplinary approach be created to make informed decisions that are consistent with the evolving situation, that NPIs be implemented, and that technological services to keep up with the daily academic activities be adopted. ${ }^{21}$

\section{Healthcare providers management}

This category includes 10 articles ${ }^{17-20} 232427293538$, among which 7 articles ${ }^{17-20} 272935$ appertain to the subcategory hospital interventions and three ${ }^{232433}$ refer to the subcategory community healthcare facilities interventions (table 2).

\section{Hospital interventions}

More than half of the articles included in this subcategory $(57 \%)^{17192732}$ reported infection screening as an effective intervention to face SARS, influenza A H1N1 and COVID-19 in Taiwan, the USA and Germany, respectively. In all articles, triage, early recognition and isolation of suspected/confirmed cases were mentioned as the most useful tools to implement this measure, followed by temperature checks $(16.6 \%)^{32}$ and the development of a nurse-driven protocol and an out-of-hospital tent $(16.6 \%) .{ }^{19}$ The use of a tent proved to be cost-effective in terms of quality of care indicators in the paediatric population during influenza A H1N1 in the USA. ${ }^{19}$ The development of a new mechanism for integrated infectious disease control, namely Traffic Control Bundling, in combination with already established intrahospital infection control interventions such as hand hygiene campaigns-alcohol dispensers coupled with hand disinfection checkpoints-proved to be effective for influenza A H1N1 and SARS outbreak containments and control in Taiwan. ${ }^{17}$ Hand hygiene, through tools like sanitisers and hand disinfection checkpoints, was reported as an effective measure by $43 \%$ of studies. ${ }^{172027}$ Two articles ${ }^{2027}$ reported the effectiveness of personal protective equipment, such as face masks and gloves, in facing respiratory infectious disease outbreaks at a healthcare provider level during the SARS and influenza A H1N1 in Taiwan. ${ }^{17} 20$ With regard to the MERS outbreak in Qatar, ${ }^{29}$ intrahospital surveillance, through dedicated technology ${ }^{17}$ and standardised protocols, ${ }^{17} 29$ was described as an important approach to managing the outbreak. A strategic communication plan was considered imperative during influenza A H1N1 in the $\mathrm{USA}^{18}$ and Canada $^{35}$ and was implemented through tools like technology, the Hospital Incident Command System (HICS) and daily conference calls. The education and training of healthcare workers, ${ }^{17} 2035$ patients and visitors, ${ }^{18} 20$ through the implementation of guidelines, was deemed an important action at the healthcare provider level during the influenza A H1N1 and SARS outbreaks in Canada and Taiwan, respectively. In a survey by Nhan et al of Canadian physicians, the need for better coordination and definition of roles and responsibilities, increased use of information technologies, merged communications and transparency in the decision-making process, increased flexibility and less contradiction in clinical practice guidelines and increased laboratory/clinical capacity were all emphasised and based on their experience during influenza A H1N1. All studies emphasised the fact that there is a need for a combination of interventions in order to be successful in responding to and containing the outbreaks.

\section{Community healthcare facilities interventions}

Three articles described the implementation of specific out-of-hospital (community) healthcare facilities dedicated to early recognition, triage, and isolation of suspected cases during influenza A H1N1 and COVID-19. ${ }^{23} 24$ In 2009, central city and 


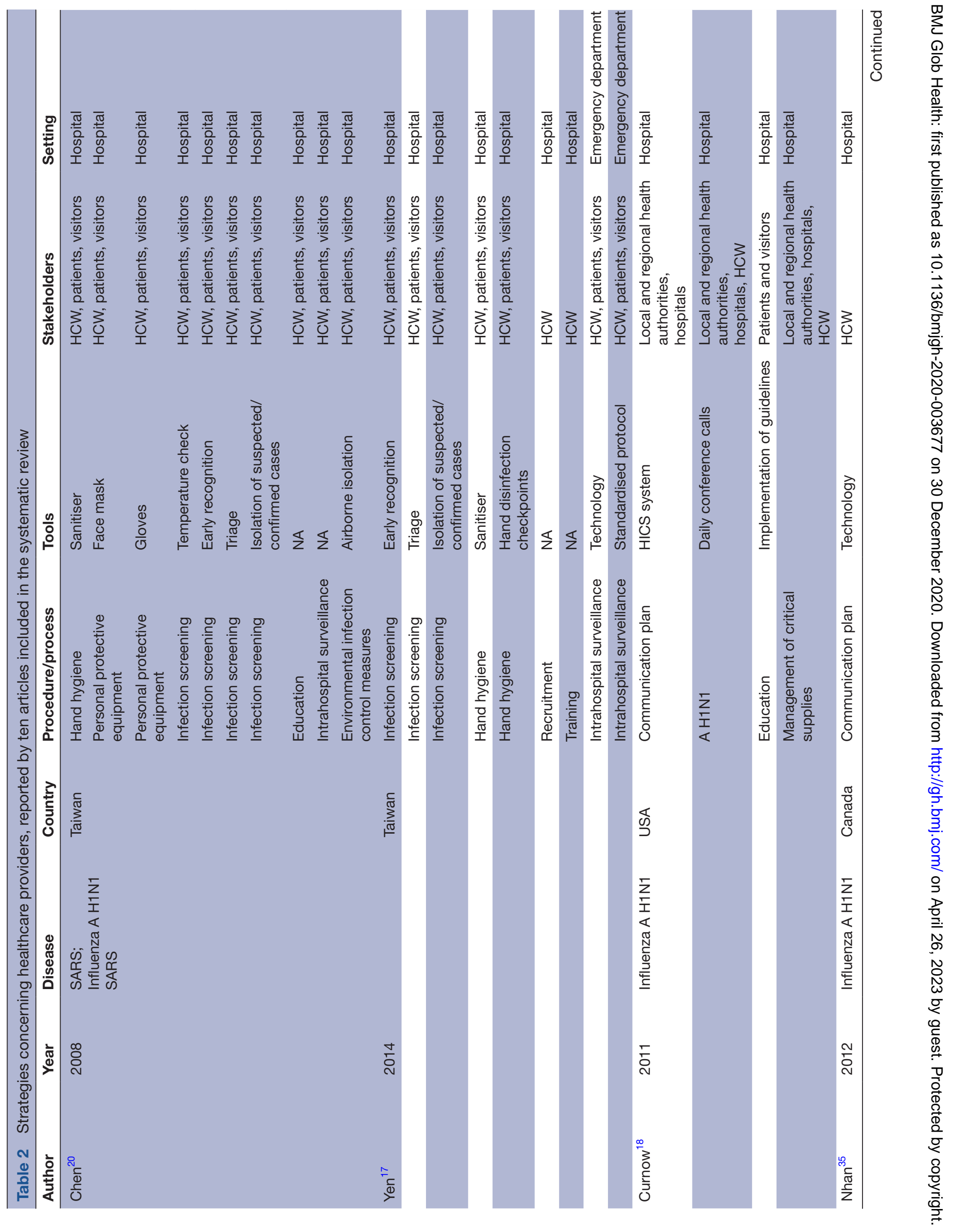




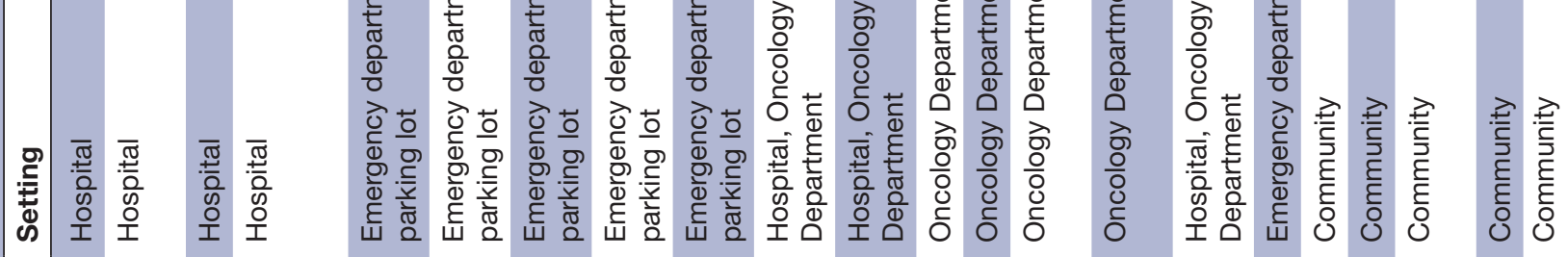
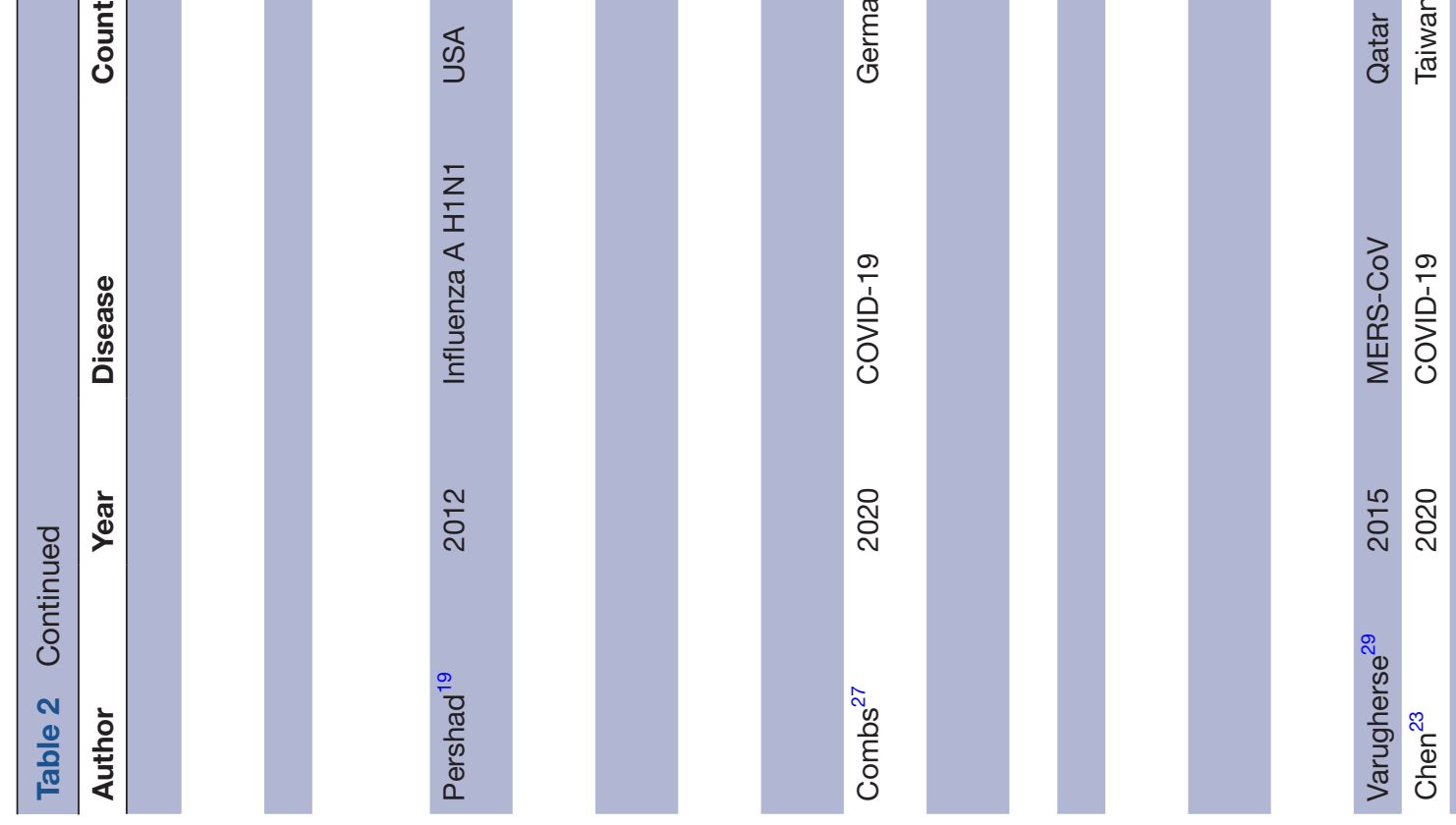

ำ

ㄴ) 융

จิ

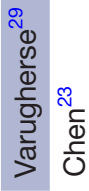

咅

స్్․ 
rural influenza centres were opened to manage the influenza A H1N1 pandemic in New Zealand ${ }^{33}$ and were aimed at working directly with the emergency departments and providing advice, diagnosis, isolation and treatments to symptomatic patients as well as postexposure prophylaxis and prompt treatment to healthcare staff, when needed. The coordinated response among the several community centres was successful in not overwhelming the hospitals and healthcare services and allowed an actively synergic intervention at a regional level. More recently, large temporary hospitals, called 'Fancang shelter hospitals', have been built by converting public venues into healthcare facilities to face the COVID-19 epidemic in Wuhan, China. ${ }^{23}$ These facilities provided isolation for suspected and confirmed cases, triage, basic medical care, frequent monitoring and rapid referral, essential living and social engagement. They proved to free up the limited medical infrastructure of higher-level hospitals and to increase the overall efficiency of care. Similar facilities, using a digital health information system, were implemented in Korea for the strict isolation and active surveillance of patients with mild symptoms, and turned out to be very costeffective and resource-saving in the management of the COVID-19 public health emergency. ${ }^{24}$

\section{DISCUSSION}

This systematic review summarised interventions and strategies implemented to approach public health critical situations across different countries over time as they related to SARS, MERS, influenza A H1N1 and COVID-19. It shows the knowledge base derived from previous experience facing the respiratory viral outbreaks. The aim of this review is to reveal what the arrival point of already occurred pandemics was, what the lessons learnt from the past are and what the scientific community should do to improve the level of evidence regarding the preparedness for future public health emergencies. The identification of these measures and their comparison over time can inform the shared approaches that are required to control the transmission of the infections as well as reduce the impact on population's health and health services.

At a national level, there is a need to develop and issue policies and guidelines for pandemic preparedness, control and management to help the decisionmaking process. This need is emphasised in the available articles describing the experience of countries such as Germany, Denmark, Finland, Mexico, UK, USA, China and Taiwan during respiratory viral infectious outbreaks. ${ }^{1722} 2628303436$ Most of these articles refer to influenza A H1N1 and SARS and, as a result, call on the importance of managing new pandemics by counting on previous experiences. These national-level policies should describe how state, tribal, local, and territorial health departments, as well as key personnel and 
institutions, should operate during a pandemic, and encourage these policies to be frequently updated. As previous experiences report, it is important for nations' management systems to be based on international cooperation and transparency, as well as on personal connections between health experts and governmental officials. ${ }^{173036}$ When facing a pandemic, all administrative levels, including international, national, local and the institutional, should be closely coordinated and interconnected, ${ }^{22} 263036$ to promote unified leadership, comprehensive coordination and hierarchical responsibility. ${ }^{22}$ Most studies pressed on the indisputable need for systematic surveillance at a national level and strengthening public health interventions. ${ }^{1722303436}$

As previously reported in the literature, the comparative evaluation of the appropriateness of national response measures during pandemics provides important lessons. Schwartz and Schwartz underlined how the Chinese experience during the outbreak of SARS determined important changes in Chinese public health system, as well as a proactive response to influenza A H1N1. ${ }^{39}$ Moreover, the USA, Mexico and Singapore undertook alert and response policies in the critical phases of the influenza A H1N1 pandemic, which contributed to China's risk-adverse strategy. ${ }^{40}$ Additionally, in contrast to other countries like the USA and Australia, Mexican and Japanese governments developed national action plans for the influenza A H1N1 pandemic, including a defined emergency decision-making task force. ${ }^{41}$ More recently, regarding public health measures implemented worldwide for COVID-19, national NPIs were deployed to cope with the first phases of pandemic across different countries (China, South Korea, Italy, Iran, France and the USA) and reported a substantial reduction of the growth rate of SARS-CoV-2 infection. ${ }^{42}$ Nevertheless, the comparative evaluation of response strategies is a major controversial issue, especially because of the uncertainty surrounding the progression of pandemics. ${ }^{41}$

The 24 articles included in this systematic review revealed the need for a coordinated and multidisciplinary response that is supported by evidence-based decisions and is employed across different sectors. Effective public health measures require cooperation among hospitals, community healthcare centres, policy makers, different workplaces, health authorities and the general public. Most of the data retrieved from the included studies reported strategies implemented at a national level, which could subsequently be adapted at a facility level. Only a few studies have reported strategies occurring at a facility level, ${ }^{15} 192327$ including workplace infection containment interventions, ${ }^{14}{ }^{15}$ community healthcare facilities, ${ }^{23} 24$ intrahospital infection containment interventions ${ }^{27}$ and organisations. ${ }^{19}$ In particular, the articles addressing the healthcare providers in specific hospitals highlighted the importance of appropriate planning and guidelines to ensure the sustainability of the hospital's response during a respiratory viral epidemic. Moreover, key components for the prevention of virus diffusion and infection control included better coordination and definition of roles and responsibilities, merged communications and transparency in the decisiona-making process, increased use of information technologies to improve communication and information among stakeholders and finally the provision of education for both patients and healthcare workers. The combination of hospital infection control measures, the development of standardised protocols, in particular for the emergency departments, and the enhanced intrahospital surveillance were reported as effective strategies in coping with the overwhelmed statuses of intrahospital departments. ${ }^{17-20} 2729323543$ An improvement in the hospital response to the emergency was seen after the building and development of out-of-hospital facilities and community healthcare facilities, which were costeffective and resource-saving during influenza A H1N1 and COVID-19 in China and Korea. The role of these facilities is to provide advice, diagnosis, isolation and treatments to symptomatic patients. ${ }^{232433}$

The healthcare system strategies described in the literature to approach respiratory viral pandemics, from outbreaks to transition phases, were based on the implementation of combined measures to enable the realisation of a coordinated response. To facilitate the comparison among studies' results, we divided these measures into different domains: workplace and university measures, NPIs, communication strategies, healthcare policy development, mental health interventions and epidemiological surveillance.

Concerning the workplaces, the combination of body temperature measurements before leaving work each day, reporting any symptoms of influenza-like illness, and an obligation to stay at home for symptomatic workers, were deemed important to minimise the disease transmission in the healthcare and nonhealthcare businesses and to enable a safe return to work. ${ }^{141521}$

NPIs were reported as fundamental to the control virus transmission in healthcare and non-healthcare environments when vaccines or specific therapeutics are not available: all of the included articles emphasised the importance of implementing multiple, combined NPIs to control influenza A H1N1, SARS, MERS and COVID19 , suggesting the continuity of these measures from the outbreak to the epidemic transition phases. Our results are also consistent with a previous review reporting the use of multiple NPIs as a crucial strategy in reducing the risk of transmission of respiratory viruses. ${ }^{44}$ Not only do NPIs reduce disease transmission, but it has been reported that they do so without necessarily further depressing economic activity. ${ }^{45}$

An effective communication strategy was also considered essential to face a pandemic: it was highlighted that public health programmes must assure an excellent communication with the public along with planning for 
effective communication within the healthcare community. Clear, trusted and coordinated communication is considered essential to avoid unnecessary risk of infection, confusion, anger, and the overwhelming demand for healthcare. ${ }^{46}$ Further, it was reported that the strategies implemented at a national level during a pandemic should consider countries' past experiences. The policy planning and development should enable the realisation of a coordinated response. The key components of a pandemic management policy included the identification of the key operators in all of the involved institutions and the development of specific guidelines and strategies to cope with limited resources. ${ }^{36}$

Additionally, cooperation among different countries, connections among community leaders and public health authorities, ${ }^{30}$ enhancement of scientific research, promotion of information exchange and the creation of international governmental networks should be considered when designing policies. Other relevant aspects reported in the literature concern strategies to address the impact of epidemics on the mental health of both healthcare workers and the general population. Thus, the aforementioned policies should pay particular attention to the integration of mental health interventions into the general deployment of disease prevention, in order to tackle the risk of negative psychological outcomes. As reported during COVID-19 in China, ${ }^{28}$ the strategies related to mental health should first consider the understanding of the psychological status of people and identify the needs of people at risk to provide appropriate interventions. During all the phases of an epidemic, a continuously enhanced surveillance programme should be put in place to contain secondary outbreaks.

The findings of this systematic review emphasised the need to continue the implementation of control measures during all phases of respiratory viral infection pandemics, and to not lower the guard during the transition phases. Until a vaccine or a specific therapy is found, healthcare systems will face the threat for a new pandemic wave that will pose continuing challenges. Therefore, healthcare systems should identify and implement the measures needed for an effective pandemic response, based also on the previous experiences. Strategies developed by various countries provide insights for all stakeholders and are important not only to ensure an effective response but also to allow rapid recovery times, and financial and political stability. ${ }^{47}$ Concerning the COVID-19, pandemic there has been little evidence of international or regional coordination, as countries close borders and look inwards. ${ }^{48}$ The current scenario has posed the need for a unified response rather than diverse, disconnected strategies. ${ }^{49}$ Regarding this, the European Union has published a roadmap ${ }^{50}$ setting out the principles that should underpin decisions to open up, noting that health policy is a national competence, but decisions should be discussed and communicated with neighbouring member states.

This systematic review fills a gap in sharing knowledge regarding the strategies that should be developed and implemented during all the phases of respiratory viral epidemics or pandemics. However, our results should be seen in the light of some weaknesses. Most of the articles included in the systematic review did not report primary data, and only $58 \%$ of the articles had a specific study design. The rest were literature reviews or guidelines that did not follow a designated methodological approach, which gives little certainty to the evidence reported. Moreover, only few primary data supporting the effectiveness of implementing specific response measures were available in the included articles. ${ }^{14} 192932$ For this reason, it was not possible to discriminate which measures were most effective in fighting epidemics. Hence, there is the need for primary data based on the evidence to guide the structuring and implementation of strategies, specifically in regard to healthcare system and healthcare provider approaches to public health emergencies like those related to respiratory viral pandemics, including COVID-19. Moreover, the data reported by the included articles mostly concerned high-income countries. Considering that the pandemic response strategies are in fact context-specific and may vary based on the level of economic development in a country, our results may not be generalisable. Furthermore, we included only articles published in English, indicating the presence of a potential publication bias. Additional articles published in other languages reporting the strategies implemented at a national level might impact our overall results. We also could have missed relevant documents from institutions or organisations that are publicly available in the grey literature, which we did not explore, since we selected only articles published in peer-reviewed journals.

This work offers an overview of recommendations, interventions and strategies based on previously experienced respiratory viral epidemics that can inform and guide the current approaches to the COVID-19 pandemic. A more useful comparison among several approaches applied worldwide would require an evidence-based reporting of information and not just qualitative or descriptive data. However, the importance of learning from the past, in order to not repeat the same mistakes, should be seen in the light of the differences between previous respiratory viral pandemics. ${ }^{51}$ In this context, the results of this systematic review suggest that public health measures should be implemented considering the circumstances of each country.

\section{CONCLUSION}

The lack of indicators and measurable outcomes is a limit for both the assessment of the implemented measures and adopted interventions, and for the evaluation of strategies and policies effectiveness related to them. A more detailed reporting would be required from 
the scientific community, to help the improvement of strategic actions addressing the epidemics and, most importantly, allowing the coordination and integration of the interventions needed to contain the pandemics. The entire community should work together to meet the challenges posed by a respiratory viral infectious disease diffusion, to achieve adequate public health preparedness.

Contributors All authors contributed to revise work for important intellectual content, gave the final approval of the version to be published, and agreed on all aspects of the work, especially concerning its accuracy and integrity. Further specific activities have been distributed as follows: FC and WR conceived the research hypothesis and designed the study. MF, IH and DZ performed the article screening, the data extraction and quality assessment. SB and MLDP reviewed the results obtained from the data extraction process considering the quality assessment of the papers. FC and WR wrote the manuscript with input from the entire team (written contributions of single paragraphs).

Funding The authors have not declared a specific grant for this research from any funding agency in the public, commercial or not-for-profit sectors.

Competing interests None declared.

Patient consent for publication Not required.

Provenance and peer review Not commissioned; externally peer reviewed.

Data availability statement Data sharing not applicable as no datasets generated and/or analysed for this study. No data are available.

Supplemental material This content has been supplied by the author(s). It has not been vetted by BMJ Publishing Group Limited (BMJ) and may not have been peer-reviewed. Any opinions or recommendations discussed are solely those of the author(s) and are not endorsed by BMJ. BMJ disclaims all liability and responsibility arising from any reliance placed on the content. Where the content includes any translated material, BMJ does not warrant the accuracy and reliability of the translations (including but not limited to local regulations, clinical guidelines, terminology, drug names and drug dosages), and is not responsible for any error and/or omissions arising from translation and adaptation or otherwise.

Open access This is an open access article distributed in accordance with the Creative Commons Attribution Non Commercial (CC BY-NC 4.0) license, which permits others to distribute, remix, adapt, build upon this work non-commercially, and license their derivative works on different terms, provided the original work is properly cited, appropriate credit is given, any changes made indicated, and the use is non-commercial. See: http://creativecommons.org/licenses/by-nc/4.0/.

ORCID iD

Fidelia Cascini http://orcid.org/0000-0001-6499-0734

\section{REFERENCES}

1 Roux J, Massonnaud C, Crépey P. COVID-19: One-month impact of the French lockdown on the epidemic burden. medRxiv 2020:2020.04.22.20075705.

2 WHO. WHO Director-General's opening remarks at the media briefing on COVID-19, 2020- 2 March. Available: https://www.who. int/dg/speeches/detail/who-director-general-s-opening-remarks-atthe-media-briefing-on-covid-19-2-march-2020

3 Coronavirus disease (COVID-19). Available: https://www.who.int/ emergencies/diseases/novel-coronavirus-2019

4 Lai S, Ruktanonchai NW, Zhou L, et al. Effect of non-pharmaceutical interventions for containing the COVID-19 outbreak in China. medRxiv 2020:2020.03.03.20029843.

5 Ferguson NM, Laydon D, Nedjati Gilani G. Impact of nonpharmaceutical interventions (NPIs) to reduce COVID19 mortality and healthcare demand.

6 Kucharski AJ, Russell TW, Diamond C, et al. Early dynamics of transmission and control of COVID-19: a mathematical modelling study. Lancet Infect Dis 2020;20:553-8.

7 Di Domenico L, Pullano G, Sabbatini CE. Currently under screening at medRxiv expected impact of lockdown in île-de-France and possible exit strategies, 2020. Available: www.epicx-lab.com/covid19.html
8 WHO. Considerations in adjusting public health and social measures in the context of COVID-19: interim guidance. Available: https:// www.who.int/publications/i/item/considerations-in-adjusting-publichealth-and-social-measures-in-the-context-of-covid-19-interimguidance

9 WHO. Strengthening and adjusting public health measures throughout the COVID-19 transition phases policy considerations for the who European region, 2020. Available: http://www.euro.who.int/ pubrequest

10 Liberati A, Altman DG, Tetzlaff J, et al. The PRISMA statement for reporting systematic reviews and meta-analyses of studies that evaluate healthcare interventions: explanation and elaboration. $B M J$ 2009;339:b2700.

11 Moola Set al. Chapter 7: Systematic Reviews of Etiology and Risk. in JBI Manual for Evidence Synthesis (JBI, 2020.

12 Baethge C, Goldbeck-Wood S, Mertens S. SANRA-a scale for the quality assessment of narrative review articles. Res Integr Peer Rev 2019;4:5.

$13 \mathrm{NIH}$. Study quality assessment tools | NHLBI, NIH. Available: https:// www.nhlbi.nih.gov/health-topics/study-quality-assessment-tools

14 Miyaki K, Sakurazawa $\mathrm{H}$, Mikurube $\mathrm{H}$, et al. An effective quarantine measure reduced the total incidence of influenza $\mathrm{A} \mathrm{H} 1 \mathrm{~N} 1$ in the workplace: another way to control the $\mathrm{H} 1 \mathrm{~N} 1$ flu pandemic. J Occup Health 2011;53:287-92.

15 Rebmann T, Wang J, Swick Z, et al. Health care versus non-health care businesses' experiences during the $2009 \mathrm{H} 1 \mathrm{~N} 1$ pandemic: financial impact, vaccination policies, and control measures implemented. Am J Infect Control 2013;41:e49-54.

16 Weeramanthri TS, Robertson AG, Dowse GK, et al. Response to pandemic (H1N1) 2009 influenza in Australia - lessons from a State health department perspective. Aust Health Rev 2010;34:477-86.

17 Yen M-Y, Chiu AW-H, Schwartz J, et al. From SARS in 2003 to H1N1 in 2009: lessons learned from Taiwan in preparation for the next pandemic. J Hosp Infect 2014;87 :185-93. vol.

18 Curnow ES, Wiles RE, Wyatt M. Lessons learned: managing a pandemic in a multihospital system. Crit Care Nurs Q 2011;34:60-7.

19 Pershad J, Waters TM. Use of tent for screening during H1N1 pandemic: impact on quality and cost of care. Pediatr Emerg Care 2012:28:229-35.

20 Chen Y-C, Chang S-C, Tsai K-S, et al. Certainties and uncertainties facing emerging respiratory infectious diseases: lessons from SARS. $J$ Formos Med Assoc 2008;107:432-42.

21 Sahu P. Closure of universities due to coronavirus disease 2019 (COVID-19): impact on education and mental health of students and academic staff. Cureus 2020;12:4-9.

22 Wang J, Wang Z. Strengths, weaknesses, opportunities and threats (Swot) analysis of china's prevention and control strategy for the covid-19 epidemic. Int J Environ Res Public Health 2020;17:2235

23 Chen S, Zhang Z, Yang J, et al. Fangcang shelter hospitals: a novel concept for responding to public health emergencies. Lancet 2020;395:1305-14.

24 Park PG, Kim CH, Heo Y, et al. Out-Of-Hospital cohort treatment of coronavirus disease 2019 patients with mild symptoms in Korea: an experience from a single community treatment center. $J$ Korean Med Sci 2020;35:2-7.

25 Shah K, Kamrai D, Mekala H, et al. Focus on mental health during the coronavirus (COVID-19) pandemic: applying Learnings from the past outbreaks. Cureus 2020;12:e7405.

26 Qualls N, Levitt A, Kanade N, et al. Community mitigation guidelines to prevent pandemic influenza - United States, 2017. MMWR Recomm Rep 2017;66:1-34

27 Combs SE, Belka C, Niyazi M, et al. First statement on preparation for the COVID-19 pandemic in large German speaking Universitybased radiation oncology departments. Radiat Oncol 2020;15:1-12.

28 Li W, Yang Y, Liu Z-H, et al. Progression of mental health services during the COVID-19 outbreak in China. Int J Biol Sci 2020:16:1732-8.

29 Varughese S, Read JG, Al-Khal A, et al. Effectiveness of the middle East respiratory syndrome-coronavirus protocol in enhancing the function of an emergency department in Qatar. Eur J Emerg Med 2015;22:316-20.

30 Ear S. Swine flu. Mexico's handling of $\mathrm{A} / \mathrm{H} 1 \mathrm{~N} 1$ in comparative perspective. Politics Life Sci 2012;31:52-66.

31 Bennett L, Waterer G. Control measures for human respiratory viral infection. Semin Respir Crit Care Med 2016;37:631-9.

32 Chu C-Y, Li C-Y, Zhang H, et al. Quarantine methods and prevention of secondary outbreak of pandemic (H1N1) 2009. Emerg Infect Dis 2010;16:1300-2.

33 Williams D, Begg A, Burgess K, et al. Influenza H1N1 2009 in Canterbury: a case study in pandemic response co-ordination. $J$ Prim Health Care 2010;2:323-9. 
34 Fleming DM, Durnall $\mathrm{H}$. Ten lessons for the next influenza pandemican English perspective: a personal reflection based on community surveillance data. Hum Vaccin Immunother 2012;8:138-45.

35 Nhan C, Laprise R, Douville-Fradet M, et al. Coordination and resource-related difficulties encountered by Quebec's public health specialists and infectious diseases/medical microbiologists in the management of $A(\mathrm{H} 1 \mathrm{~N} 1)$--a mixed-method, exploratory survey. BMC Public Health 2012;12:115.

36 Krumkamp R, Ahmad A, Kassen A, et al. Evaluation of national pandemic management policies-A hazard analysis of critical control points approach. Health Policy 2009;92:21-6.

37 Charania NA, Tsuji LJS. The 2009 H1N1 pandemic response in remote first nation communities of subarctic Ontario: barriers and improvements from a health care services perspective. Int $J$ Circumpolar Health 2011;70:564-75.

38 Williams D, Begg A, Burgess K, et al. Influenza H1N1 2009 in Canterbury: a case study in pandemic response co-ordination. $J$ Prim Health Care 2010;2:323-9.

39 Schwartz RD, Schwartz J. Confronting Global Pandemics : Lessons from China and the U. S. 1-21, 2003.

40 Xue L, Zeng GA. Comprehensive Evaluation on Emergency Response in China - The Case of Pandemic Influenza (H1N1) 2009 2019;253.

41 Xue L, Zeng G. Global strategies and response measures to the influenza A (H1N1). Pandemic 2019:15-44.

42 Hsiang S, Allen D, Annan-Phan S, et al. The effect of large-scale anti-contagion policies on the COVID-19 pandemic. Nature 2020;584:262-7.
43 World Health Organisation. Hospital preparedness for epidemics. 71, 2014.

44 Smith SMS, Sonego S, Wallen GR, et al. Use of nonpharmaceutical interventions to reduce the transmission of influenza in adults: a systematic review. Respirology 2015;20:896-903.

45 Correia S, Luck S, Verner E. Pandemics depress the economy, public health interventions do not: evidence from the $1918 \mathrm{flu}$. SSRN Journal 2020.

46 Levin PJ, Gebbie EN, Qureshi K. Can the health-care system meet the challenge of pandemic flu? Planning, ethical, and workforce considerations. Public Health Rep 2007;122 :573-8. vol..

47 Blanchard K, Ragupathy D. Are we there yet? the transition from response to recovery for the COVID-19 pandemic. Progress in Disaster Science 2020;7:100102.

48 Martin McKee: The questions we need to answer before easing lockdown - The BMJ. Available: https://blogs.bmj.com/bmj/2020/05/ 06/martin-mckee-the-questions-we-need-to-answer-before-easinglockdown/

49 Forman R, Atun R, McKee M, et al. 12 lessons learned from the management of the coronavirus pandemic. Health Policy 2020;124:577-80. vol..

50 European Commission. Coronavirus: Commission roadmap shows path towards. Available: https://ec.europa.eu/commission/ presscorner/detail/en/ip 20652

51 Kucharski A, Barrie F. The rules of contagion: why things SpreadAnd why they stop, 2020. 